\title{
Optimal Policy and the Risk Properties of Human Capital Reconsidered
}

\author{
Dan Anderberg* \\ Royal Holloway University of London, IFS, CEPR, and CESifo
}

October 23, 2008

\begin{abstract}
This paper considers how optimal education and tax policy depends on the risk properties of human capital. It is demonstrated that a key feature of human capital investments is whether they increase or decrease wage risk. In a benchmark model it is shown that this feature alone determines whether a constrained optimal allocation should be characterized by a positive or a negative education premium. In the same model a positive intertemporal wedge is optimal. A set of generalizations, including non-observability of education, nonobservability of consumption, and temporal resolution of uncertainty, are then considered to examine the robustness of these results.
\end{abstract}

Keywords: Education, Optimal Taxation, Risk

JEL Classification: D81, H21, I21

${ }^{*}$ I would like to thank two anonymous referees for a number of very useful comments. All remaining errors are my own. 


\section{Introduction}

For an individual contemplating investing in education there are numerous sources of uncertainty. She is likely to be imperfectly aware of her own abilities, the quality of the education she is considering, the probability of succeeding etc. Moreover, further uncertainties await her when she enters the labor market: unemployment shocks, fluctuations in demand etc. imply that, even conditional on obtaining a certain degree, her position in the earnings distribution may be far from certain.

The question of whether, empirically, human capital investments are risky was addressed already in Becker (1964) who noted that there was more wage variation among college graduates than among high school graduates. More recent empirical work however questions whether this reflects a true increase in wage uncertainty. Two main issues make inferring the relation between education and wage risk problematic. First, individuals are unlikely to be completely ignorant about their abilities etc. Hence, one should be careful to distinguish between "heterogeneity" and "uncertainty" - only what is unknown to the individual at the time of the investment decision should be considered as uncertainty. Second, individuals self-select into education; this implies that the variance of wages among college graduates may not be a reliable guide to what would have been the variance of wages among actual high school graduates had they decided to go to college etc.

Recent empirical work has taken on the challenge of trying to separate out heterogeneity from uncertainty and to deal with selection. Carneiro, Hansen, and Heckman (2003) and Cunha, Heckman and Navarro (2005) estimate models where the individuals choose between high school and college, and where the unobserved component of an individual's earnings has a factor structure. By estimating how the various factors relate to potential earnings, what the factor distributions are, and which factors where known to the individual when making the decision, the authors separate heterogeneity from uncertainty while effectively also accounting for selection. Chen (2008) takes a different approach, combining a parametric selection model with an instrumental variable approach. Results from both approaches confirm that investments in education are associated with substantial genuine uncertainty. E.g. based on their findings, 
Cunha, Heckman and Navarro (2005, p. 253) argue that if individuals knew their ex post earnings outcomes resulting from their schooling options, a substantial fraction $(25-30 \%)$ would change their schooling decisions. While it is thus clear that individuals do face substantial uncertainty regarding their returns to education, what is also important is whether wage uncertainty increases or decreases with the level of education. While the aforementioned contributions seem to suggest that wage uncertainty may be increasing between high school and college (see e.g. Chen, p. 285, Fig. 1), it is fair to say that more evidence is needed before a consensus on this point can be reached.

Policy interventions in the area of education are also widespread and mostly designed to encourage young individuals to invest more in human capital. The policy tools used tend to combine mandatory school leaving age policies with subsidy policies. Perhaps not surprisingly then there is also a small theoretical literature on the role of the risk properties of human capital for optimal tax- and education policy. In a seminal paper, Eaton and Rosen (1980) showed that introducing a redistributive linear income tax improves welfare when the return to education is stochastic. Hamilton (1987), extending the analysis, showed that some policy instrument to encourage education, e.g. a capital income tax, would be a desirable complement to an income $\operatorname{tax}$.

Three more recent contributions have focused on tax and education policy in environments entirely characterized by uncertainty $\left.\right|^{1}$ Grochulski and Piskorski (2006) consider nonlinear optimal taxation in a dynamic model where education is endogenous and is not observable to the government. They find that a constrained optimal allocation is characterized by a positive education premium and by a positive intertemporal wedge. There are two reasons for the latter wedge. First, higher future consumption makes it difficult to achieve self-selection (Diamond, 2006). Second, a lower (implicit) return to savings provides incentive to invest in human capital; this is optimal since the tax on earnings discourages education due to its cost not being deductible. da Costa and Maestri (2007) consider nonlinear optimal income taxation in a twoperiod model where education is observable to the government. In addition to considering the

\footnotetext{
${ }^{1}$ See also Anderberg and Andersson (2003) for an early contribution.
} 
constrained efficient level of education, the authors also consider what policy tools are required to implement an efficient allocation. da Costa and Maestri also demonstrate the optimality of a positive intertemporal wedge; in contrast, they claim that a zero education premium is generally optimal. Jacobs, Schindler and Yang (2008) consider a similar two-period economy, but assume a linear income tax. Moreover, their focus is not on characterizing the second-best optimal level of education, but on characterizing an optimal linear net education subsidy. One main result in their study is that the optimal education subsidy is higher the more complementary are education and labor supply. This highlights how, in the linear income tax framework, an optimal education subsidy, by boosting investments in education and hence labor supply incentives, is designed to mitigate the distortionary effect of the income tax. They also show how an optimal education subsidy corrects for a fiscal externality that obtains when the income tax provides partial insurance (see Section II below).

Parallel to the literature on wage uncertainty and optimal policy is a literature that focuses on optimal tax- and education policy under heterogeneity only. This literature thus assumes that the individuals are fully informed about their potential earnings at all levels of education. This literature includes contributions by Bovenberg and Jacobs (2005), Bohacek and Kapicka (2008), Maldonado (2008), and Jacobs and Bovenberg (2008) .

The aim of the current paper is to synthesize and extend the literature on optimal policy in the pure uncertainty framework. E.g. we reconsider the characterization of a constrained efficient level of education provided by da Costa and Maestri (2007); we demonstrate how the results of Grochulski and Piskorski (2006) on the optimality of an education premium depends on their assumed form for the wage risk; moreover, we relate our results to those obtained by Jacobs, Schindler and Yang (2008) by relating our characterization of the constrained optimal level of education to an optimal (implicit) education subsidy. We also extend the literature by considering the roles played by the process through which individuals learn their idiosyncratic earnings components, and by unobserved intertemporal trades. Furthermore, we demonstrate that the model with uncertainty only has strong parallels to models that focus exclusively on heterogeneity. 
The outline of the paper is as follows. Section II sets up and analyzes a benchmark model. Section III generalizes the model by considering non-observability of education, unobserved intertemporal trades, and the process of resolution of uncertainty. Section IV concludes.

\section{A Benchmark Model}

There is a continuum of unit measure of individuals who live for two periods, $t=0,1$. Each individual has one unit of time to allocate in each period and obtains utility from consumption and disutility from labor and education. 2 Preferences are additively separable within periods, $u\left(c_{t}\right)+v\left(l_{t}+z_{t}\right)$, where $c_{t}$ is consumption, $l_{t}$ is labor supply, and $z_{t}$ is time devoted to education. $u(\cdot)$ increasing, concave, and continuously differentiable while $v(\cdot)$ is decreasing, concave and continuously differentiable. Utility is also additive across periods and the individual discounts utility at rate $\beta \in(0,1]$.

Education only occurs in the first period $\left(z_{1} \equiv 0\right)$ and we let $z$ denote the amount of education undertaken at $t=0$. Moreover, the first period is devoted entirely to education and labor supply $\left(l_{0} \equiv 1-z\right)$. An individual's effective labor supply per unit of time worked at $t=0$ is $w_{0}$. Her productivity in the second period depends on education $z$ and on an idiosyncratic productivity shock $\theta$. There is a discrete set of shocks, $\theta \in \Theta=\left\{\theta_{1}, \ldots, \theta_{N}\right\}$, ordered increasingly. The effective labor supply per unit of time worked at $t=1$ is then $w(z, \theta)$, which is assumed to be strictly increasing in both arguments, and continuously differentiable and strictly concave in $z$. Each individual's productivity shock $\theta$ is an independent draw from a probability distribution with density $\pi$. The p.d.f. $\pi(\cdot)$ is common knowledge. An individual only learns her productivity shock $\theta$ at the beginning of $t=1$, and this information then becomes private information.

Aggregate output depends on inputs of capital, $k_{t}$, and aggregate effective labor supply, $Y_{t}$. The production technology is, for simplicity, taken to be linear: $F\left(k_{t}, Y_{t}\right)=k_{t}+Y_{t}$ with

$$
Y_{0} \equiv w_{0}(1-z), \quad \text { and } \quad Y_{1} \equiv \sum_{\theta \in \Theta} y_{1}(\theta) \pi(\theta)
$$

\footnotetext{
${ }^{2}$ The basic model is the same as in da Costa and Maestri (2007) except we allow for any finite number of shocks.
} 
where $y_{1}(\theta) \equiv w(z, \theta) l_{1}(\theta)$ is the effective labor supply for an individual of type $\theta$ at $t=1$. The economy is endowed with $k_{0} \geq 0$ units of capital which are taken as given. The economy is assumed to be competitive in the standard sense, implying that $w_{0}$ and $w(z, \theta)$ also represent the individuals' hourly wage rates in the two periods.

There is no heterogeneity among individuals at $t=0$ and all consume a common amount $c_{0}$; in contrast, at $t=1$, consumption $c_{1}(\theta)$ etc. generally varies across types. Given the linearity of the technology the resource constraint can be consolidated across periods and written as

$$
c_{0}+\sum_{\theta \in \Theta} \pi(\theta)\left[c_{1}(\theta)-y_{1}(\theta)\right] \leq k_{0}+w_{0}(1-z) .
$$

Note that the marginal rate of transformation across the two periods is unity.

\section{The Unconstrained Optimal Allocation}

Consider first the case where the social planner can observe individual types and can perfectly control each individual's consumption and labor supply. Suppose also that the social planner maximizes ex ante expected utility. The planner's problem can be written as

$$
\max _{\mathbf{c}, \mathbf{y}, z}\left\{u\left(c_{0}\right)+\beta \sum_{\theta \in \Theta} \pi(\theta)\left[u\left(c_{1}(\theta)\right)+v\left(y_{1}(\theta) / w(z, \theta)\right)\right] \text { s.t. (2) }\right\},
$$

where the vectors $\mathbf{c}$ and $\mathbf{y}$ describe all relevant consumptions and effective labor supplies. At the optimum, consumption is fully insured against the idiosyncratic shock so that $c_{1}$ is independent of $\theta$; moreover consumption satisfies a standard Euler equation $u^{\prime}\left(c_{0}\right)=\beta u^{\prime}\left(c_{1}\right)$. Optimal labor supply, $l_{1}(\theta)$, is increasing in $\theta$. Of special interest to us is the characterization of the efficient human capital investment,

$$
\sum_{\theta \in \Theta} \pi(\theta) l_{1}(\theta) w_{z}(z, \theta)=w_{0}
$$

which can be written more succinctly as $E\left(l w_{z}\right)=w_{0}$. This first-best rule balances the marginal cost in terms of foregone production at $t=0$ with the marginal benefit of expected higher productivity at $t=1$.

As we will see below, the first-best rule (4) will generally not hold when the agents have private information about their productivity shocks. We will then say that there is a "positive education premium" if $E\left(l w_{z}\right)>w_{0}$ and a "negative education premium" if $E\left(l w_{z}\right)<w_{0}$. 


\section{The Constrained Efficient Allocation}

Consider now the case where the productivity shocks obtained by the agents are not observed by the planner. The planner is, however, assumed to observe any intertemporal trades that the individuals might attempt to undertake as well as the individuals' educational investments. This implies that the planner can also observe individual consumption at both dates. Furthermore, as in a standard Mirrlees (1971) model, the planner does not observe the wage (since $\theta$ is private information) and not hours of work, but does observe the product of the two, i.e. gross earnings $y$.

Relying on the revelation principle, efficient allocations can be characterized by assuming that the planner uses a direct mechanism where the individuals report their types $\theta$ at $t=1$, and chooses an allocation $(\mathbf{c}, \mathbf{y}, z)$. Given the use of a direct mechanism, an individual chooses a reporting strategy $\sigma$ which is a mapping from $\Theta$ into itself. We use $\Sigma$ to denote the set of all possible reporting strategies. The truthtelling strategy is denoted $\sigma^{*}$. We will also be making frequent use of the set

$$
I_{\theta}^{\sigma} \equiv\left\{\theta^{\prime} \in \Theta \mid \sigma\left(\theta^{\prime}\right)=\theta\right\}
$$

This is the set of types $\theta^{\prime}$ at which, given strategy $\sigma$, the individual reports $\theta$. Given the allocation $(\mathbf{c}, \mathbf{y}, z)$, the expected utility of the agent when adopting reporting strategy $\sigma$ is

$$
W(\sigma) \equiv u\left(c_{0}\right)+\beta \sum_{\theta \in \Theta} \pi(\theta)\left[u\left(c_{1}(\sigma(\theta))\right)+v\left(y_{1}(\sigma(\theta)) / w(z, \theta)\right)\right]
$$

An allocation $(\mathbf{c}, \mathbf{y}, z)$ is said to be incentive compatible if $W\left(\sigma^{*}\right) \geq W(\sigma)$ for all $\sigma \in \Sigma$ and it is said to be constrained efficient if it maximizes expected utility among all incentive compatible and resource feasible allocations.

Definition $1 A$ constrained efficient allocation $(\mathbf{c}, \mathbf{y}, z)$ is a solution to

$$
\max _{\mathbf{c}, \mathbf{y}, z}\left\{W\left(\sigma^{*}\right) \mid W\left(\sigma^{*}\right) \geq W(\sigma) \text { for all } \sigma \in \Sigma \text { and (2) }\right\} \text {. }
$$

\section{Properties of the Constrained Efficient Allocation}

The unconstrained optimal allocation characterized above will not be incentive compatible some incentive constraint must strictly bind at the constrained optimum. Moreover, it can be 
shown that the only reporting strategies for which there can be binding incentive constraints are those where the agent does not over-report any shock $3^{3}$ In other words, any reporting strategy for which the incentive constraint binds will be element of the set $\Sigma_{0} \subset \Sigma$ defined as

$$
\Sigma_{0} \equiv\{\sigma \in \Sigma \mid \sigma(\theta) \leq \theta \text { for all } \theta \in \Theta\}
$$

Consider now the first order conditions for the planner's problem in this benchmark case. For first- and second period consumption we obtain,

$$
u^{\prime}\left(c_{0}\right)=\lambda, \quad \text { and } \quad u^{\prime}\left(c_{1}(\theta)\right)\left\{1+\sum_{\sigma \in \Sigma} \phi_{\sigma}\left[1-\sum_{\theta^{\prime} \in I_{\theta}^{\sigma}} \frac{\pi\left(\theta^{\prime}\right)}{\pi(\theta)}\right]\right\}=\frac{\lambda}{\beta},
$$

where $\lambda$ is the multiplier on the resource constraint (2) and $\phi_{\sigma}$ is the multiplier on the incentive constraint for reporting strategy $\sigma$. Using these two equations, we can consider whether there is an optimal intertemporal wedge. Indeed, combining the two equations shows that the "inverse Euler equation" holds at the optimum $4^{4}$

$$
\frac{1}{u^{\prime}\left(c_{0}\right)}=\frac{1}{\beta} \sum_{\theta \in \Theta} \frac{\pi(\theta)}{u^{\prime}\left(c_{1}(\theta)\right)} .
$$

The upshot from this, which follows from the application of Jensen's inequality, is that the

\footnotetext{
${ }^{3}$ In brief the argument, which follows Guesnerie and Seade (1982), goes as follows. Since the agents take no actions until $t=1$, the planner's problem can be separated into two stages. The first stage involves setting $c_{0}$ and $z$; the second stage problem involves choosing $\left\{\mathbf{c}_{1}, \mathbf{y}_{1}\right\}$ and is a standard Mirrleesian optimal income tax problem with a finite set of types and an implicitly utilitarian social welfare function. Hence any property of the optimum of the second stage problem that holds for any given $z$ and $c_{0}$ will also apply at the overall constrained optimum. The optimum of the second stage problem, for any $z$ and $c_{0}$, involves a sequence of bundles $\left(c_{1}^{i}, y_{1}^{i}\right)$, $i=1, \ldots, K$ (with $K<N$ if there is bunching at some point and $K=N$ otherwise) which can be ordered increasingly: $j>i$ implies $\left(c_{1}^{j}, y_{1}^{j}\right) \gg\left(c_{1}^{i}, y_{1}^{i}\right)$. With a utilitarian objective and with separable preferences, a resource feasible downward redistribution of consumption, $d c_{1}^{i+1}<0$ and $d c_{1}^{i}>0$, would be welfare improving. Any such redistribution must therefore violate incentives. In other words, each pair of successive points must be linked together by some type $\theta \in \Theta$ who is indifferent between $\left(c_{1}^{i+1}, y_{1}^{i+1}\right)$ and $\left(c_{1}^{i}, y_{1}^{i}\right)$ and who is allocated the higher of the two bundles. Since the agents' preferences satisfy "single-crossing" it follows that, conversely, each type $\theta$ strictly prefers her equilibrium bundle to all higher bundles in the sequence.

${ }^{4}$ The inverse Euler equation is now routine in the literature and is discussed e.g. in Golosov, Tsyvinski and Werning (2006).
} 
allocation is distorted towards early consumption. We refer to this as a "positive intertemporal wedge" (Golosov, Tsyvinski and Werning, 2006).

Proposition 1 (Positive intertemporal wedge). At the constrained efficient allocation there is a positive intertemporal wedge, $u^{\prime}\left(c_{0}\right)<\beta \sum_{\theta \in \Theta} \pi(\theta) u^{\prime}\left(c_{1}(\theta)\right)$.

The intuition for why a positive intertemporal wedge is desirable is straightforward. An agent who deviates by under-reporting her productivity shock would not only work less, but would also have less consumption than under truthtelling; with additive preferences this results in a higher marginal utility of consumption and hence a greater incentive to save. Then, making savings less available relaxes the incentive constraints (see e.g. Diamond and Mirrlees (1978), Golosov, Kocherlakota and Tsyvinski (2003), and Diamond (2006)).

To have some short-hand for labor supplies under an arbitrary reporting strategy, define $l_{1}^{\sigma}(\theta) \equiv y_{1}(\sigma(\theta)) / w(z, \theta)$. We simply use $l_{1}(\theta)$ to denote the labor supply at $\theta$ under truthtelling. The first order condition for effective labor supplies can then be written as

$$
-\frac{v^{\prime}\left(l_{1}(\theta)\right)}{w(z, \theta)}\left\{1+\sum_{\sigma \in \Sigma} \phi_{\sigma}\left[1-\sum_{\theta^{\prime} \in I_{\theta}^{\sigma}} \frac{\pi\left(\theta^{\prime}\right)}{\pi(\theta)} \frac{v^{\prime}\left(l_{1}^{\sigma}\left(\theta^{\prime}\right)\right)}{v^{\prime}\left(l_{1}(\theta)\right)} \frac{w(z, \theta)}{w\left(z, \theta^{\prime}\right)}\right]\right\}=\frac{\lambda}{\beta} .
$$

By combining (11) and (9) the optimal intra-temporal wedge at $t=1$ can be studied. However, the planner's problem at $t=1$ (given the optimal $c_{0}$ and $z$ ) is, as noted above, a standard Mirrleesian optimal tax problem, which implies that the constrained efficient allocation here will inherit all the standard properties. E.g. it can be shown that there is a strictly positive implicit marginal income tax rate at all incomes $y_{1}(\theta)$ except for the highest type, for whom the implicit marginal tax rate is zero (Guesnerie and Seade, 1982).

More interesting for us is the characterization of the optimal human capital investment. The first order condition for $z$ can, after collecting terms, be written as

$$
-\sum_{\theta \in \Theta} \pi(\theta) v^{\prime}\left(l_{1}(\theta)\right) l_{1}(\theta)\left\{1+\sum_{\sigma \in \Sigma} \phi_{\sigma}\left[1-\frac{v^{\prime}\left(l_{1}^{\sigma}(\theta)\right) l_{1}^{\sigma}(\theta)}{v^{\prime}\left(l_{1}(\theta)\right) l_{1}(\theta)}\right]\right\} \frac{w_{z}(z, \theta)}{w(z, \theta)}=\frac{\lambda}{\beta} w_{0} .
$$

This equation can be further manipulated by extending it and using (11); we then obtain the following characterization of the constrained efficient level of education:

$$
\sum_{\theta \in \Theta} \pi(\theta) l_{1}(\theta) w_{z}(z, \theta)-w_{0}=\frac{\beta}{\lambda} A
$$


with

$$
A \equiv \sum_{\sigma \in \Sigma} \phi_{\sigma} \sum_{\theta \in \Theta} \pi(\theta) v^{\prime}\left(l_{1}^{\sigma}(\theta)\right) l_{1}^{\sigma}(\theta)\left\{\frac{w_{z}(z, \sigma(\theta))}{w(z, \sigma(\theta))}-\frac{w_{z}(z, \theta)}{w(z, \theta)}\right\} .
$$

Note that the first-best rule for $z$ (i.e. $\left.E\left(l w_{z}\right)=w_{0}\right)$ holds at the constrained optimum if and only if $A=0$. If $A>0$, then $E\left(l w_{z}\right)>w_{0}$ at the constrained optimum implying that there is a positive education premium. Conversely, if $A<0$, then $E\left(l w_{z}\right)<w_{0}$, i.e. there is a negative education premium.

Since the only binding incentive constraints are for $\sigma$ where $\sigma(\theta) \leq 0$ for all $\theta$ (with some strict inequality) we see that $A$ can be signed if $w_{z} / w$ is monotonic in $\theta$. In particular, there will, at the constrained optimum, be a positive education premium if $w_{z} / w$ is strictly increasing in $\theta$ and negative education premium if $w_{z} / w$ is strictly decreasing in $\theta !^{5}$

Monotonicity of $w_{z} / w$ in $\theta$ has at least two empirical interpretations. First, note that $w_{z} / w$ being increasing (decreasing) in $\theta$ is equivalent to the education elasticity of the wage being increasing (decreasing) in $\theta$. The empirical content of this clearly depends on how one interprets the idiosyncratic productivity factor $\theta$. Second, note that $w_{z} / w$ is the derivative of the log wage with respect to education; from this it is easy to show that if $w_{z} / w$ is increasing (decreasing) in $\theta$, then the variance of the log wage increases (decreases) in education $\sqrt[6]{6}$

For this reason we will say that "education increases wage risk" if $w_{z} / w$ strictly increases in $\theta$, and we will say that "education decreases wage risk" if $w_{z} / w$ strictly decreases in $\theta$. If $w_{z} / w$ is independent of $\theta$ then we will say that "education is neutral with respect to wage risk".

We can now summarize the result regarding the optimal level of education.

Proposition 2 (Socially optimal education premium). If education increases (decreases) wage risk then, at the constrained efficient allocation, there is a strictly positive (negative) education

\footnotetext{
${ }^{5}$ As noted by Maldonado (2008), $w_{z} / w$ can be either increasing or decreasing in $\theta$ even if we were to impose weak complementarity of $z$ and $\theta$ in the wage function, i.e. if we assume $w_{z \theta} \geq 0$. What matters is whether $z$ and $\theta$ are complements or substitutes in the log wage.

${ }^{6}$ Differentiating the identity $\operatorname{var}(\ln w \mid z) \equiv E\left(\ln w^{2} \mid z\right)-E(\ln w \mid z)^{2}$ (with expectation taken over $\left.\theta\right)$ with respect to $z$ yields $2 E\left[(\ln w-E(\ln w \mid z))\left(w_{z} / w\right) \mid z\right]$. The result then follows from the fact that $\ln w-E(\ln w \mid z)$ is, given $z$, an increasing function of $\theta$ which has zero expected value. The empirical relationship between education and the variance of log wages has recently been considered by Chen (2008).
} 
premium. If education is neutral with respect to wage risk, then the education premium at the constrained efficient allocation is zero.

We would expect that a distortion in education is introduced in order to relax incentive constraints. Indeed, this is the case. One way to see this is to note that the marginal "informational rent" (i.e. utility gain) accruing to type $\theta_{i+1}$ from being this type rather than being type $\theta_{i}$ can be written as

$$
v\left(\frac{y_{1}\left(\theta_{i}\right)}{w\left(z, \theta_{i+1}\right)}\right)-v\left(\frac{y_{1}\left(\theta_{i}\right)}{w\left(z, \theta_{i}\right)}\right) \approx-v^{\prime}\left(l_{1}\left(\theta_{i}\right)\right) l_{1}\left(\theta_{i}\right)\left(1-\frac{w\left(z, \theta_{i}\right)}{w\left(z, \theta_{i+1}\right)}\right)>0
$$

An decrease in $z$ decreases this informational rent precisely when $w_{z} / w$ is increasing in $\theta$.

When will the constrained efficient allocation be characterized by a zero education premium, i.e. when will the first-best rule obtain at the social optimum? A functional form commonly adopted in the literature is the multiplicative wage function $w(z, \theta)=w(z) \gamma(\theta)$; for this particular form it is easy to see that $w_{z} / w$ is independent of $\theta$.

COROLlary 3 If the wage function $w(z, \theta)$ has a multiplicative form, $w(z, \theta)=w(z) \gamma(\theta)$ for some increasing functions $w(\cdot)$ and $\gamma(\cdot)$, then the education premium at the constrained efficient allocation is zero.

The benchmark model considered here is the same as that presented by da Costa and Maestri (2007), extended from two to $N$ shocks. These authors, however, claim that the first-best rule for $z$ holds at the constrained efficient allocation irrespective of the form for $w(z, \theta)$ (da Costa and Maestri (2007), p. 701). The above results suggest that their claim is erroneous and would appear to be due to an algebraic error.

\section{Implicit Net Subsidies to Education}

The literature on optimal education and tax policy tends to focus on two related but separate issues. The first is how the second-best optimal level of education relates to the first-best characterization. This was the issue considered in the analysis above. The second is whether 
eduction is (implicitly or explicitly) subsidized at the social optimum 7 In order to clarify the connection between these two issues we consider here, for the benchmark model, the question of whether education is implicitly subsidized or taxed at the social optimum. We should be careful to stress that the implicit subsidies or taxes on education and savings that we derive here will generally not implement the constrained efficient allocation as linear instruments will typically not be sufficiently powerful 8 The purpose of the analysis is rather to relate the optimality of an implicit net tax/subsidy to the optimality of an education premium, as well as to previous results in the literature.

To this end consider a typical agent who faces an income tax $T(\cdot)$ at $t=1$. At $t=0$ the agent chooses the educational investment $z$ and how much to save $s$; then at $t=1$, after having observed $\theta$, the agent decides on her labor supply. The agent is facing a linear $\operatorname{tax} \tau$ on earnings at $t=0$. A positive $\operatorname{tax} \tau>0$ acts as an implicit subsidy to education since it lowers its cost (which is entirely in terms of foregone earnings). Savings are taxed at rate $\chi$ leading to the gross return $1-\chi$. For simplicity we assume that the agent's private educational costs (in net present value form), $w_{0}(1-\tau)(1-\chi) z$, are deductible from gross earnings when calculating the taxable earnings at $t=1$. This implies that the total effective marginal subsidy to education is adjusted ex post to compensate for the realized marginal income tax rate. We can then interpret any $\tau>0$ as a net subsidy to education.

The agent's problem can then be written as

$$
\begin{array}{cc}
\max _{z, s, \mathbf{c}, 1} & u\left(c_{0}\right)+\beta \sum_{\theta \in \Theta} \pi(\theta)\left[u\left(c_{1}(\theta)\right)+v\left(l_{1}(\theta)\right)\right] \\
\text { s.t. } & c_{0}=w_{0}(1-\tau)(1-z)-s \\
\text { and } & c_{1}(\theta)=w(z, \theta) l_{1}(\theta)+s(1-\chi)-T\left(y_{1}^{n}(\theta)\right) \\
\text { with } & y_{1}^{n}(\theta)=w(z, \theta) l_{1}(\theta)-w_{0}(1-\tau)(1-\chi) z
\end{array}
$$

\footnotetext{
${ }^{7}$ Recent contributions that consider the optimality of education subsidies in the presence of either linear or nonlinear income taxation include Bovenberg and Jacobs (2005), Maldonado (2008), Jacobs and Bovenberg (2008), Bohacek and Kapicka (2008), Jacobs, Schindler and Yang (2008). The first four consider environments where the individuals know their idiosyncratic earnings components from the outset and hence face no uncertainty. The last model considers an environment similar to that considered here but assumes a linear income tax.

${ }^{8}$ See da Costa and Maestri (2007) for a discussion of implementation in the current environment.
} 
In this formulation $y_{1}^{n}(\theta)$ represents the agent's taxable earnings at $t=1$ in state $\theta$.

Two equations characterize the agent's choice of $s$ and $z$. The intertemporal allocation of consumption satisfies the standard Euler equation

$$
u^{\prime}\left(c_{0}\right)=\beta(1-\chi) \sum_{\theta \in \Theta} \pi(\theta) u^{\prime}\left(c_{1}(\theta)\right)
$$

In order to characterize the agent's choice of $z$ it is useful to define

$$
\alpha(\theta) \equiv u^{\prime}\left(c_{1}(\theta)\right)\left[1-T^{\prime}\left(y^{n}(\theta)\right)\right], \quad \text { and } \quad \rho_{z} \equiv-\frac{\operatorname{cov}\left(\alpha, l_{1} w_{z}\right)}{E(\alpha) E\left(l_{1} w_{z}\right)}
$$

where $\alpha(\theta)$ is the agent's ex post marginal utility of gross earnings, and where we refer to $\rho_{z}$ as the "risk-premium" for $z$. We then obtain the following characterization of the agent's choice of $z$,

$$
\sum_{\theta \in \Theta} \pi(\theta) l_{1}(\theta) w_{z}(z, \theta)=\frac{w_{0}(1-\tau)(1-\chi)}{1-\rho_{z}} .
$$

This shows that, as expected, either a tax on first period earnings $\tau>0$, or a tax on savings $\chi>0$, encourages investments in education. Moreover, even if $\tau=\chi=0$, the agent will deviate from the first-best rule $E\left(l_{1} w_{z}\right)=w_{0}$ if $\rho_{z} \neq 0$; if the gross return to the investment, $l_{1} w_{z}$, is high when the marginal utility of gross earnings $\alpha$ is low, then $\rho_{z}>0$, implying a lower investment. This is the rational response by the agent to risk (Levhari and Weiss, 1974).

Combining (19) with the characterization of the constrained efficient level of education in (13) and (14) yields that the optimal (implicit) net subsidy rate on education satisfies

$$
\frac{(1-\tau)(1-\chi)}{1-\rho_{z}}-1=\frac{\beta}{\lambda w_{0}} A .
$$

From Proposition 1 and equation (17) it follows that savings are implicitly taxed, $\chi>0$, at the constrained optimum. Suppose first that $w_{z} / w$ is independent of $\theta$, implying that $A=0$ and that the first-best rule for $z$ obtains at the constrained efficient optimum. Solving for the implicit optimal education subsidy rate then yields

$$
\tau=\tau^{0} \equiv \frac{\rho_{z}-\chi}{1-\chi}
$$

which is generally different from zero for two reasons. First, when $\rho_{z}>0$, the agent is holding back the educational investment due to its risky nature. While this is a rational response to 
risk by the agents, in the presence of an optimal income tax it implies an underinvestment from a social point of view ${ }^{9}$ Second, even if $\rho_{z}=0$, the positive tax on savings is already encouraging education; hence the optimal education subsidy counteracts this effect. If $w_{z} / w$ is not independent of $\theta$ it then follows from equation (20) and the discussion of the previous section that the optimal $\tau$ is less (larger) than $\tau^{0}$ if education increases (decreases) wage risk.

Jacobs, Schindler and Yang (2008) derive an optimal education subsidy rate for a similar economic environment with risky investments in education, but assuming a linear income tax. In addition to highlighting the role of the risk-premium, $\rho_{z}$, these authors stress the role of the complementarity between education and labor supply. This is natural since, with a linear income tax, the issue is not one of relaxing self-selection constraints, but rather one of boosting labor supply in order to mitigate the distortionary effect of the income tax.

There is also a strong connection between the results obtain here with risky investments in education and results obtained in models with only heterogeneity and no uncertainty. E.g. Maldonado (2008) presents a model which is effectively a static version of the above benchmark model, except the agents know $\theta$ from the outset and there are only two types (with $\theta$ interpreted as "ability"). Education can, in that environment, vary across types. Nevertheless, Maldonado derives the result that the education level for the mimicked lower type should be distorted downwards (upwards) relative to the first-best rule if $w_{z} / w$ increases (decreases) in $\theta 10$ Similarly, he shows that the mimicked low type should then face a net tax (subsidy) on education.

Maldonado's result has recently been generalized by Jacobs and Bovenberg (2008) who consider a general earnings function $y=\Phi(z, l, \theta)$ and a continuum of types 11 They show that education should be subsidized on a net basis as $\varepsilon_{l z}>\varepsilon_{\theta z}$ and taxed when the opposite inequality

\footnotetext{
${ }^{9}$ See Jacobs, Schindler and Yang (2008) for an in-depth discussion.

${ }^{10}$ Indeed, while Maldonado prefers to present this main result in the form of his equation (15), he could equally well have presented it in the following form,

$$
l^{L} w_{z}^{L}-1=\frac{\mu}{\lambda} \frac{\pi^{H}}{\pi^{L}} v^{\prime}\left(\widehat{l}^{H}\right) \widehat{l}^{H}\left[\frac{\widehat{w}_{z}^{H}}{\widehat{w}^{H}}-\frac{w_{z}^{L}}{w^{L}}\right]
$$

where $L$ and $H$ refers to the low- and high type respectively, and where "hat" indicate values for the mimicker i.e. the high type reporting being low type. This is the direct counterpart of our equations $(13)$ and $(14)$.

${ }^{11}$ See also Bohacek and Kapicka (2008).
} 
applies, where $\varepsilon_{k z} \equiv\left(\Phi \Phi_{k z}\right) /\left(\Phi_{z} \Phi_{k}\right), k=l, \theta$, is the Hicks' partial elasticity of complementarity. Complementarity between $z$ and $l$ calls for a subsidy to education: the intuition is that education then encourages labor supply and hence reduces the distortionary cost of taxation. In contrast complementarity between ability and education calls for a net tax on education since education increases the earnings gap between ability groups (a negative equity effect). When $\varepsilon_{\theta z}=\varepsilon_{l z}$ the two effects precisely cancel out. Furthermore, when $y=l w(z, \theta)$ it follows that $\varepsilon_{l z}=1$ while $\varepsilon_{\theta z}=1$ precisely when $w_{z} / w$ is independent of $\theta$.

\section{Generalizations and Robustness}

Two main results were obtained for the constrained efficient allocation in the benchmark economy. (i) If education increases (decreases) wage risk, there should be a positive (negative) education premium. (ii) The inverse Euler equation holds, implying a positive intertemporal distortion.

In this section we will explore the robustness of these two results by considering a number of generalizations to the benchmark model. In particular, we will consider the roles played by (i) observability of the educational investments, (ii) observability of intertemporal trades and consumption, and (iii) the process by which the individuals learn their idiosyncratic productivity components.

The importance of the relationship between education and wage risk for the constrained optimal level of education is shown to be general. As long as education is observable by the planner any deviation from the first-best efficiency rule comes about as the result of education either increasing or decreasing wage risk. When education is not observable, the wage risk effect is still operating, but the non-observability causes a second independent downward distortion in the optimal level of investment.

The structure of the optimal intertemporal wedge depends heavily on all three factors considered. Non-observability of education makes a case for a positive intertemporal wedge in order to encourage human capital investments. A positive intertemporal wedge is also desirable when the agents are yet to receive some private information; conversely, once $\theta$ has been fully revealed 
to the agents the justification of a positive intertemporal wedge as a mechanism to elicit private information disappears. Non-observability of consumption generally also impacts on the optimal intertemporal wedge, and one can even construct cases where the optimal wedge is negative.

We will treat the cases of unobservable investments in education and unobservable intertemporal trades separately. We will, however, introduce a general model for how the agents learn their idiosyncratic productivity shocks that we maintain throughout.

\section{Modelling the Temporal Resolution of Uncertainty}

We will assume throughout that no information about $\theta$ is available when the level of education is decided. The opposite case where $\theta$ is known to the agents at the outset - i.e. when there is only heterogeneity and no uncertainty - has recently been studied by several authors ${ }^{12}$ We will however consider the possibility that the agents learn something (or possibly even everything) about $\theta$ prior to completing their consumption at date $t=0$. This captures the idea that, plausibly, individuals make their education decisions earlier than their savings decisions, and hence may have more information when taking the latter decision.

To model the temporal resolution of uncertainty we assume that, at the end of period $t=0$, each individual obtains a signal $\mu \in M$ which may be informative about $\theta$. We use $\pi(\mu)$ to denote the probability of signal $\mu$, and we use $\pi(\mu, \theta)$ and $\pi(\theta \mid \mu)$ to denote the joint- and conditional probabilities of shocks and signals. One simple interpretation of this setup is as follows. Students differ in scholastic ability and productivity shocks, with the two dimensions of heterogeneity being correlated; however, they only learn their abilities while in education, e.g. through privately observed test scores. The benchmark model above was a special where the agents received no information (as e.g. in the case where $|M|=1$ ). A second special case is that where the signal is "fully revealing"; in this we take it that $M=\Theta$ and $\pi(\theta \mid \mu=\theta)=1$ ).

In the analysis below we will maintain the assumption that the planner implements a direct mechanism. This implies that consumption at $t=0$ can depend on the signal $\mu$ whereas consumption etc. at time $t=1$ can depend on both the signal $\mu$ and the final realization of the

\footnotetext{
${ }^{12}$ See Bovenberg and Jacobs (2005), Maldonado (2008), Jacobs and Bovenberg (2008), Bohacek and Kapicka (2008).
} 
idiosyncratic shock $\theta$. A reporting strategy $\sigma \in \Sigma$ now maps $M$ into $M$ at time $t=0$ (how to report signals) and $M \times \Theta$ into $M \times \Theta$ at $t=1$ (how to report at each node $(\mu, \theta)$ ).

Extending definition (5) above, we now let

$$
I_{\mu}^{\sigma} \equiv\left\{\mu^{\prime} \in M \mid \sigma\left(\mu^{\prime}\right)=\mu\right\} \text {, and } I_{\mu, \theta}^{\sigma} \equiv\left\{\left(\mu^{\prime}, \theta^{\prime}\right) \in M \times \Theta \mid \sigma\left(\mu^{\prime}, \theta^{\prime}\right)=(\mu, \theta)\right\}
$$

Here $I_{\mu}^{\sigma}$ is the set of signals at which, under reporting strategy $\sigma$, the agent reports that the signal is $\mu . I_{\mu, \theta}^{\sigma}$ has the same interpretations but for final nodes rather than for signals.

\section{Unobservable Investments in Education}

We will first consider the case where the agents' investments in education are not observable by the planner. Since the cost of education is assumed to be entirely in the form of foregone earnings, we assume that first-period earnings are not observable 13 We assume however that any savings done by an agent, as well as gross earnings at $t=1$, are observable by the planner. Hence individual consumption at this date is observable.

The $z$ chosen by the planner should be thought of as an intended (or "recommended") level of investment. The agents can make unobserved deviations, $\Delta_{z}$, from this level. Note that any such deviation has to be chosen by the agent without knowledge of either $\mu$ or $\theta$. Defining $c_{0}(\mu)$ as the level of consumption enjoyed at time $t=0$ by an agent who reports signal $\mu$ and who does not deviate from $z$, the actual consumption of the agent when deviating from $z$ by $\Delta_{z}$ is then $c_{0}(\mu)-w_{0} \Delta_{z}$.

\section{Individual Behavior}

Consider then the expected utility for an agent who adopts reporting strategy $\sigma$ and deviates from $z$ by $\Delta_{z}$. This expected utility is given by

$W\left(\sigma, \Delta_{z}\right) \equiv \sum_{\mu \in M} \pi(\mu)\left\{u\left(c_{0}(\sigma(\mu))-w_{0} \Delta_{z}\right)+\beta \sum_{\theta \in \Theta} \pi(\theta \mid \mu)\left[u\left(c_{1}(\sigma(\mu, \theta))\right)+v\left(\frac{y_{1}(\sigma(\mu, \theta))}{w\left(z+\Delta_{z}, \theta\right)}\right)\right]\right\}$.

\footnotetext{
${ }^{13}$ If we had modelled other components to the cost of education, such as direct costs, then we could have maintained observability of first-period earnings. Hence this assumption is merely a simplification.
} 
Given any $\sigma \in \Sigma$ the agent will choose the education deviation optimally. Hence define $\Delta_{z}(\sigma)$ as the solution to the problem $\max _{\Delta_{z}} W\left(\sigma, \Delta_{z}\right)$ and, somewhat abusing the notation, define $W(\sigma) \equiv W\left(\sigma, \Delta_{z}(\sigma)\right)$ as the expected utility of choosing reporting strategy $\sigma$.

\section{Efficient Allocations}

When characterizing efficient allocations we can restrict our attention to allocations where the agents report signals and shocks truthfully and do not deviate from the recommended educational investment. We can then write the generalized resource constraint as

$$
\sum_{\mu \in M} \pi(\mu)\left\{c_{0}(\mu)+\sum_{\theta \in \Theta} \pi(\theta \mid \mu)\left[c_{1}(\mu, \theta)-y_{1}(\mu, \theta)\right]\right\} \leq k_{0}+w_{0}(1-z) .
$$

The planner's problem which defines a constrained efficient allocation now looks as follows.

Definition $2 A$ constrained efficient allocation $(\mathbf{c}, \mathbf{y}, z)$ is a solution to

$$
\max _{\mathbf{c}, \mathbf{y}, z}\left\{W\left(\sigma^{*}, 0\right) \mid W\left(\sigma^{*}, 0\right) \geq W(\sigma) \text { for all } \sigma \in \Sigma \text { and (24) }\right\} \text {. }
$$

The first order conditions associated with problem (25) are direct generalizations of those for the benchmark model. However, since they are somewhat notationally involved, we relegate them to an appendix.

In the benchmark model it was argued that the incentive constraints would only ever bind for reporting strategies where the agent does not over-report any $\theta$. We would expect this to be the normal case also in generalized versions of the model. Hence we will assume that the same holds in this extended environment. To formalize this, let $\sigma_{\theta}(\mu, \theta)$ denote the productivity shock reported at node $(\mu, \theta)$ under $\sigma \in \Sigma$. We will then assume that if the incentive constraint binds for $\sigma$ in problem (25) then $\sigma \in \Sigma_{0} \subset \Sigma$, where, generalizing definition (8) above,

$$
\Sigma_{0} \equiv\left\{\sigma \in \Sigma \mid \sigma_{\theta}(\mu, \theta) \leq \theta \text { for all }(\mu, \theta) \in M \times \Theta\right\}
$$

It is also natural to assume that an agent who plans to under-report her productivity will also deviate downwards in terms of her educational investment. This can be proven to be the case when there are no signals. The logic is simple. Self-selection implies that earnings in period $t=1$ are increasing in $\theta$. An agent who plans to under-report her productivity at some $\theta$ 
will then supply less labor than under truthtelling, and will hence have less incentives to invest in education 14 Since the logic can be expected to hold more generally, we will assume that $\Delta_{z}(\sigma) \leq 0$ for all $\sigma \in \Sigma_{0}$.

Consider first the question of whether an intertemporal distortion is socially desirable. From the first order conditions for consumption, we can derive the following equation linking the marginal utilities,

$$
\frac{1}{u^{\prime}\left(c_{0}(\mu)\right)}=\frac{1}{\beta} \sum_{\theta \in \Theta} \frac{\pi(\theta \mid \mu)}{u^{\prime}\left(c_{1}(\mu, \theta)\right)}+\sum_{\sigma \in \Sigma} \frac{\phi_{\sigma}}{\lambda} \sum_{\mu^{\prime} \in I_{\mu}^{\sigma}} \frac{\pi\left(\mu^{\prime}\right)}{\pi(\mu)} \frac{\left[u^{\prime}\left(c_{0}(\mu)\right)-u^{\prime}\left(c_{0}(\mu)-w_{0} \Delta_{z}(\sigma)\right)\right]}{u^{\prime}\left(c_{0}(\mu)\right)} .
$$

By noting that the last term is non-negative under the assumption that $\Delta_{z}(\sigma) \leq 0$ for all binding $\sigma$ we see that a positive intertemporal wedge is still optimal.

Proposition 4 (Positive intertemporal wedge with unobservable investments in education). At the constrained efficient allocation there is a non-negative intertemporal wedge at all signals, $u^{\prime}\left(c_{0}(\mu)\right) \leq \beta \sum_{\theta \in \Theta} \pi(\theta \mid \mu) u^{\prime}\left(c_{1}(\mu, \theta)\right)$ for all $\mu \in M$.

To see the forces behind this result it is instructive to consider two special cases. First, consider the case where education is observable, represented here by $\Delta_{z}(\sigma) \equiv 0$ for all $\sigma$; then the standard inverse Euler equation would hold at every signal. This is the standard effect that a positive intertemporal wedge relaxes future incentive constraints. Consider then the case where the signal is fully revealing. In that case it is easy to see that $u^{\prime}\left(c_{0}(\theta)\right) \leq \beta u^{\prime}\left(c_{1}(\theta)\right)$ for all $\theta \in \Theta$ only due to $\Delta_{z}(\sigma) \leq 0$ for binding $\sigma$. Hence in this case the positive intertemporal wedge is entirely motivated by the non-observability of the educational investment. While Proposition 4 notes that the intertemporal wedge (at signal $\mu$ ) is non-negative, it will be strictly positive if $\mu$ is not fully revealing (so that $\pi(\cdot \mid \mu)$ has a non-degenerate support) and/or if $\Delta_{z}(\sigma)<0$ for some binding $\sigma$.

To summarize, there are two forces causing an optimal positive intertemporal wedge. (i) Nonrevealing signals: when the agents are still to receive further information about $\theta$ an inverse Euler

\footnotetext{
${ }^{14}$ In the case of fully revealing signals a sufficient condition for $\Delta_{z}(\sigma) \leq 0$ for all $\sigma \in \Sigma_{0}$ is that $y_{1}(\theta)$ and $c_{1}(\theta)$ both be increasing in $\theta . c_{1}(\theta)$ being increasing implies that the deviating agent also faces higher marginal cost of education due to having lower consumption.
} 
equation applies. (ii) Non-observability of the educational investment: when a plan to underreport future productivity shocks is accompanied by an under-investment in education, a positive intertemporal wedge implicitly encourages education, thus relaxing the incentive constraints.

Grochulski and Piskorski (2006) present a dynamic optimal tax model with unobservable investments in education. The difference between their model and that presented here is that Grochulski and Piskorski allow for multiple working periods but assume a more restrictive specification of the human capital technology $[15$ Concerning the intertemporal wedge at $t=0$, Grochulski and Piskorski highlight how the two forces mentioned above imply the optimality of a positive wedge. Proposition 4, while only considering one working period, extends their finding by showing that optimality of a positive intertemporal wedge is robust to the form of the human capital technology as well as to the process by which the agents learn their productivity realizations.

Consider now the constrained optimal investment in education. From the first order conditions we obtain the following direct generalization of the result from the benchmark model.

$$
\sum_{(\mu, \theta) \in M \times \Theta} \pi(\mu, \theta)\left[l_{1}(\mu, \theta) w_{z}(z, \theta)\right]-w_{0}=\frac{\beta}{\lambda} A
$$

with

$$
A \equiv \sum_{\sigma \in \Sigma} \phi_{\sigma} \sum_{(\mu, \theta) \in M \times \Theta} \pi(\mu, \theta) v^{\prime}\left(l_{1}^{\sigma}(\mu, \theta)\right) l_{1}^{\sigma}(\mu, \theta)\left\{\begin{array}{c}
\left(\frac{w_{z}\left(z, \sigma_{\theta}(\mu, \theta)\right)}{w\left(z, \sigma_{\theta}(\mu, \theta)\right)}-\frac{w_{z}(z, \theta)}{w(z, \theta)}\right) \\
+\left(\frac{w_{z}(z, \theta)}{w(z, \theta)}-\frac{w_{z}\left(z+\Delta_{z}(\sigma), \theta\right)}{w\left(z+\Delta_{z}(\sigma), \theta\right)}\right)
\end{array}\right\} .
$$

Note how (28) and $(29)$ generalizes $(13)$ and $(14)$; the benchmark model is the special case where there are no signals and $\Delta_{z}(\sigma) \equiv 0$.

A zero education premium would obtain if $A$ were equal to zero. The term $A$ however has two components, neither of which will generally be equal to zero. First, just as in the benchmark model, it matters whether education increases or decreases wage risk: if education increases (decreases) wage risk, this contributes positively (negatively) to $A$.

\footnotetext{
${ }^{15}$ In the model of Grochulski and Piskorski there are $T \geq 1$ working periods. The idiosyncratic productivity factor is binary at any point in time, $\theta_{t} \in\{0,1\}$ for all $t$, but $\theta=0$ is an absorbing state. Moreover, the worker's productivity at time $t$ is $w_{t}=w\left(z \theta_{t}\right)$. When $T=1$, their model is a special case of that presented here in that it assumes a particular wage function (and no signals).
} 
The second component of $A$ is non-negative under the assumption that $\Delta_{z}(\sigma) \leq 0$ for all binding $\sigma$. This follows since concavity of $w(z, \theta)$ in $z$ implies that $w_{z} / w$ decreases in $z$. Hence non-observability of education causes a generic downward distortion in education.

Proposition 5 (Socially optimal education premium with unobservable investments in education). If education either increases wage risk or is neutral with respect to wage risk then, at the constrained efficient allocation, there is a positive education premium. There can be a negative education premium at the constrained efficient allocation if education sufficiently decreases wage risk.

The above result clarifies a result obtained by Grochulski and Piskorski (2006). In their model the constrained efficient allocation is always characterized by a positive education premium. That finding is reconciled with that in the above proposition by noting that their chosen specification implies that education increases wage risk ${ }^{16}$ Their fact that they obtain an unambiguous result thus depends on the stochastic structure that they assume.

Why does non-observability of education cause a generic downward distortion in education? As pointed out by Grochulski and Piskorski, the fact that education is non-observable implies that an increase in the recommended level of education $z$ need not be adhered to by the agents. Indeed, we know that an agent who plans to misreport her productivity type will also want to underinvest in education. The larger is $z$ the larger is the scope for such deviations. Holding back $z$ hence relaxes the incentive-selection constraints by reducing the scope for double deviations involving under-reporting and under-investing.

\section{Unobservable Intertemporal Trades}

We now relax the assumption that the planner can observe individual consumption. Specifically, we introduce a market where the individuals can trade consumption intertemporally at an equilibrium price $R$. The consumption allocated by the social planner serves then only as a effective "endowments". Our modelling of a retrading market follows Golosov and Tsyvinski (2007) who

\footnotetext{
${ }^{16}$ Recall that Grochulski and Piskorski assume that $w=w(z \theta)$ with $\theta \in\{0,1\}$ (see fn. above 15). Then note that, for any $z, w_{z} /\left.w\right|_{\theta=1}>w_{z} /\left.w\right|_{\theta=0}=0$.
} 
assume that intertemporal trades are enforceable but not observed by the planner. We use the notation $x$ for allocated consumption by the planner and $c$ for actual consumption.

\section{Individual Behavior}

Since the signal $\mu$ is realized before intertemporal trades take place, the individual chooses a contingent plan for how to trade at each realization of $\mu$. Moreover, as before, she chooses a reporting strategy $\sigma \in \Sigma$. Consider the expected utility of an agent who chooses reporting strategy $\sigma$ and savings plan s. This expected utility can be written as:

$$
\begin{gathered}
W(\sigma, \mathbf{s} ; R) \equiv \sum_{\mu \in M} \pi(\mu)\left\{u\left(c_{0}(\mu)\right)+\beta \sum_{\theta \in \Theta} \pi(\theta \mid \mu)\left[u\left(c_{1}(\mu, \theta)\right)+v\left(\frac{y_{1}(\sigma(\mu, \theta))}{w(z, \theta)}\right)\right]\right\} \\
\text { with } \quad c_{0}(\mu)=x_{0}(\sigma(\mu))-s(\mu) \text { and } c_{1}(\mu, \theta)=x_{1}(\sigma(\mu, \theta))+R s(\mu) .
\end{gathered}
$$

Given any $\sigma \in \Sigma$ the agent will choose the savings plan optimally. Hence, somewhat abusing the notation, define $W(\sigma ; R)=\max _{\mathbf{s}} W(\sigma, \mathbf{s} ; R)$ (and we use $s^{\sigma}(\mu ; R)$ to denote the savings at signal $\mu$ given $\sigma$ ). It is easy to see that, in this environment, the agent's consumption plans will always follow standard Euler equations (one for each signal).

Equilibrium in the retrading market requires that aggregate savings be zero.

DEFINITION 3 An equilibrium in the retrading market given an allocation $(\mathbf{x}, \mathbf{y}, z)$ is a return $R$, a reporting strategy $\sigma \in \Sigma$, and a savings plan $\mathbf{s}$ such that (i) $\sigma$ solves $\max _{\sigma \in \Sigma} W(\sigma ; R)$ and (ii) aggregate savings are zero, $\sum_{\mu \in M} \pi(\mu) s^{\sigma}(\mu ; R)=0$.

\section{Efficient Allocations}

Relying on the revelation principle, we can focus on allocations that induce truthful reporting and where, in equilibrium, the individuals do not trade. Hence a natural characterization of an efficient allocation is that it maximizes the expected utility $W\left(\sigma^{*}, \mathbf{0} ; R^{*}\right)$ subject to this expected utility being no less than $W\left(\sigma ; R^{*}\right)$ for all $\sigma \in \Sigma$ (and subject to a resource constraint) with $R^{*}$ being the equilibrium return induced by the allocation. Golosov and Tsyvinski (2007) show, however, that this problem can be rewritten in a more convenient form in which the return $R$ appears as a choice variable for the planner. The logic behind this has similarities to 
the first-order approach in moral hazard problems where one can add a further choice variable to the principal's problem if one imposes the first-order condition characterizing the individual rational choice as a constraint, here represented by the Euler equations. Hence, rather than restating the equivalence result of Golosov and Tsyvinski, we use the following definition of a constrained efficient allocation directly:

Definition $4 A$ constrained efficient allocation $(\mathbf{x}, \mathbf{y}, z)$ along with an equilibrium return $R^{*}$ is a solution to

$$
\begin{array}{ccc}
\max _{\left(\mathbf{x}, \mathbf{y}, z, R^{*}\right)} & W\left(\sigma^{*}, \mathbf{0} ; R^{*}\right) & \\
\text { s.t. } & W\left(\sigma^{*}, \mathbf{0} ; R^{*}\right) \geq W\left(\sigma ; R^{*}\right) & \left(\phi_{\sigma}\right) \\
& u^{\prime}\left(x_{0}(\mu)\right)=\beta R^{*} \sum_{\theta \in \Theta} \pi(\theta \mid \mu) u^{\prime}\left(x_{1}(\mu, \theta)\right) & \left(\eta_{\mu} \pi(\mu)\right) \\
\text { and } & \text { 24) } \text { with } \mathbf{c}=\mathbf{x} . & (\lambda)
\end{array}
$$

The requirement that an agent who reports truthfully should not wish to retrade is ensured by the Euler equations holding at zero savings. We will continue to focus on the "normal case" where the binding incentive constraints are for reporting strategies that under-report the productivity shock, i.e. for $\sigma \in \Sigma_{0}$ as defined in (26) above.

Consider first the rule for the constrained efficient level of education. From the first order conditions we again obtain equation (28) but this time the term $A$ takes the simpler form

$$
A \equiv \sum_{\sigma \in \Sigma} \phi_{\sigma} \sum_{(\mu, \theta) \in M \times \Theta} \pi(\mu, \theta) v^{\prime}\left(l_{1}^{\sigma}(\mu, \theta)\right) l_{1}^{\sigma}(\mu, \theta)\left\{\frac{w_{z}\left(z, \sigma_{\theta}(\mu, \theta)\right)}{w\left(z, \sigma_{\theta}(\mu, \theta)\right)}-\frac{w_{z}(z, \theta)}{w(z, \theta)}\right\} .
$$

Given the assumption that the incentive constraints will only ever bind for $\sigma \in \Sigma_{0}$, Proposition 2 and Corollary 3 from the benchmark model continue to hold. Thus the role played by the relationship between education and wage risk is unaffected by the non-observability of consumption (and by the process by which the agents learn their productivity realizations).

Consider next the optimal intertemporal wedge. For this we need the optimality condition for $R$, which can be written as

$$
\sum_{\mu \in M} \pi(\mu)\left\{\sum_{\sigma \in \Sigma} \phi_{\sigma} s^{\sigma}\left(\mu ; R^{*}\right) u^{\prime}\left(c_{0}^{\sigma}(\mu)\right)+\beta R^{*} \eta_{\mu} \sum_{\theta \in \Theta} \pi(\theta \mid \mu) u^{\prime}\left(c_{1}(\mu, \theta)\right)\right\}=0,
$$

where $c_{0}^{\sigma}(\mu)=x_{0}(\sigma(\mu))-s^{\sigma}\left(\mu ; R^{*}\right)$ is the period-0 consumption at signal $\mu$ of an agent who adopts reporting strategy $\sigma$. There are two terms. The first comes from the fact that 
deviators will generally trade consumption intertemporally. Hence if savings are positive at binding reporting strategies, then lowering the return $R$ will relax these incentive constraints. The second term comes from the fact that $R^{*} \neq 1$ leads to distorted Euler equations for the truthtelling agent. Since it is difficult to say anything concrete about the optimal $R$ at this level of generality we proceed by considering a number of special cases.

No Signals In this special case there is no heterogeneity among the agents, neither in terms of "endowments" (i.e. consumption allocated by the social planner) nor in terms of beliefs about future productivity shocks, at the time when the retrading market is open. Hence the unique equilibrium entails zero trade. Assuming that the allocated consumption $x_{1}(\theta)$ is strictly increasing in $\theta$, a strictly positive intertemporal wedge can be shown to be optimal, confirming a result in da Costa and Maestri (2007) ${ }^{17}$ The result obtains from the fact that an individual will, under any non-truthtelling $\sigma \in \Sigma_{0}$, engage in strictly positive savings.

Proposition 6 (Positive intertemporal wedge with unobservable consumption and no signals) Suppose the individuals do not receive any informative signals about their productivity shocks; then $R^{*}<1$ at the efficient allocation, implying $u^{\prime}\left(c_{0}\right)<\beta \sum_{\theta \in \Theta} \pi(\theta) u^{\prime}\left(c_{1}(\theta)\right)$.

Fully Revealing Signals In the opposite extreme case all individuals learn their productivity shocks prior to taking their savings decisions. The individuals are in this case heterogenous when the retrading market is open. Nevertheless, the result, which effectively follows from Werning (2007), is that there should be no intertemporal distortions ${ }^{18}$ The intuition for this is simple: once $\theta$ has been revealed, the agents receive no further private information. Hence from that point onwards each individual will obtain some equilibrium utility from consumption which must

\footnotetext{
${ }^{17}$ Conditional on all agents choosing $s=0$, the ex post self-selection constraint are also satisfied; single-crossing then implies that $x_{1}(\theta)$ must be weakly increasing. The assumption that $x_{1}(\theta)$ is strictly increasing then simply amounts to assuming that there is no "bunching". The result then follows from 33 after noting that the multiplier $\eta$ takes the same sign as $R^{*}-1$.

${ }^{18}$ Werning (2007) considers a dynamic optimal tax problem and shows that a zero intertemporal distortion is optimal when the skill distribution is fixed across dates and states. Constancy per se is, however, not important; what matters is that the individuals obtain no further private information.
} 
be delivered at lowest resource cost, implying a zero intertemporal distortion.

Proposition 7 (Zero intertemporal wedge with unobservable consumption and revealing signals) Suppose that all individuals learn their productivity shocks prior to finalizing their consumption plans; then $R^{*}=1$ at the efficient allocation, implying $u^{\prime}\left(c_{0}(\theta)\right)=\beta u^{\prime}\left(c_{1}(\theta)\right)$ for all $\theta \in \Theta$.

Some Two-by-Two Cases The two above extreme informational scenarios produced a positive and a zero intertemporal wedge respectively. Does this mean that $R^{*} \leq 1$ is always optimal? And more generally, does non-observability of consumption have a major impact on the optimal wedge? In order to explore cases with partial information, suppose there are only two shocks $\left\{\theta_{L}, \theta_{H}\right\}$ and two signals. To simplify further, we consider two special cases: (i) Positive Revealing Signal: A fraction $\alpha$ of the agents who receive the positive productivity shock $\theta_{H}$ learn this prior to completing their consumption plans, and (ii) Negative Revealing Signal: A fraction $\alpha$ of the agents who receive the negative productivity shock $\theta_{L}$ learn this prior to completing their consumption plans. For each case we provide a numerical example using standard functional forms, $u(c)=c^{1-\delta} /(1-\delta)$ and $v(l)=\log (1-l)$. We treat $z$ (and hence wages) as given, setting $w_{0}(1-z)=0.25, w\left(z, \theta_{L}\right)=1, w\left(z, \theta_{H}\right)=3$, and $\pi\left(\theta_{L}\right)=\pi\left(\theta_{H}\right)=0.5$. We ignore discounting and initial capital, and, to sharpen the contrast in the examples, we assume a low risk aversion, $\delta=0.5$.

Consider first a positive revealing signal. For this case it can be shown that there are two binding incentive constraints. One is a standard constraint that an agent who receives the (less positive) ambiguous signal may lie about a subsequent positive productivity shock. This deviating agent would save a positive amount. The second binding constraint is that an agent who obtains the revealing favorable signal may hide this and claim a positive productivity "surprise". This deviating agent would save a negative amount since she knows that she will obtain $\theta_{H}$. Hence, the optimal intertemporal wedge may actually be either positive or negative. This is highlighted in the left panel of Figure 1 For comparison, the figure also shows the (average) optimal $R$ for the same economy but with observable consumption ${ }^{19}$ More generally,

\footnotetext{
${ }^{19}$ This is the benchmark economy extended only to allow for signals. In this economy there will be one implicit
} 
in this case, the possibility of hidden intertemporal trades has a moderating effect on the optimal wedge.

Consider then a negative revealing signal. In this case it is easy to see that no deviator will choose positive savings, implying that a positive intertemporal wedge is always optimal. The optimality of a positive intertemporal wedge is highlighted in the right panel of Figure 1. In this case the possibility of hidden intertemporal trades has a negligible impact on the optimal wedge.

\section{Conclusions}

This paper has reconsidered the optimal design of education- and redistributive policies in an environment where individuals face idiosyncratic wage risk. We paid particular attention to two questions.

The first question was whether, at a constrained efficient allocation, there should be a positive or negative education premium. We showed that this depends on whether education increases or decreases wage risk. This finding was found to be robust both to the potential existence of a hidden market where agents can retrade consumption intertemporally, as well as to the process by which the individuals learn about their idiosyncratic wage components. The exception to the rule was when education is not observable. In that case how education relates to wage risk still influences the constrained optimal level of education. However, non-observability of education also causes a second generic downward distortion in the level of human capital investments (Grochulski and Piskorski, 2006).

The second question was whether, at the constrained efficient allocation, there should be a positive intertemporal wedge. The findings here can be summarized as follows. If the agents cannot engage in intertemporal trades, then the optimal intertemporal wedge is always nonnegative; indeed, it is strictly positive as long as education is not observable (Grochulski and Piskorski, 2006) and/or the individuals have still not fully learned their idiosyncratic wage

components. If the agents can engage in hidden intertemporal trades, a zero intertemporal $R^{*}$ for each signal (which is zero at any revealing signal). Hence the $R^{*}$ highlighted is a weighted average. 
wedge is still optimal once the individuals are fully informed about their wage shocks and a positive intertemporal wedge is optimal if they are completely uninformed. However, there can be inbetween cases with partial information where even a negative intertemporal wedge can be optimal.

The main message of the current paper is thus that the empirical risk properties of education - i.e. how wage risk depends on the level of education and how individuals learn about their idiosyncratic wage components - play a key role for optimal education/tax policy. These risk properties are the focus of current empirical research. Hence in future research we hope to combine theoretical models of the type presented here with the growing empirical evidence in order to obtain empirically-based policy prescriptions. However, doing so also require extending current theory to consider cases where there is both uncertainty and individual heterogeneity at the time when the individuals make their educational decisions. 


\section{References}

Anderberg, D. \& Andersson, F. (2003), 'Investments in human capital, wage uncertainty, and public policy', Journal of Public Economics 87, 1521-1537.

Becker, G. S. (1964), Human Capital, Columbia University Press, New York.

Bohacek, R. \& Kapicka, M. (2008), 'Optimal human capital policies', Journal of Monetary Economics 55, 1-16.

Bovenberg, A. L. \& Jacobs, B. (2005), 'Redistribution and education subsidies are Siamese twins', Journal of Public Economics 89, 2005-2035.

Carneiro, P., Hansen, K. \& Heckman, J. J. (2003), 'Estimating distributions of treatment effects with an application to the returns to schooling and measurement of the effects of uncertainty on college choice', International Economic Review 44(2), 361-422.

Chen, S. H. (2008), 'Estimating the variance of wages in the presence of selection and unobserved heterogeneity', Review of Economics and Statistics 90, 275-289.

Cunha, F., Heckman, J. J. \& Navarro, S. (2005), 'Separating uncertainty from heterogeneity in life cycle earnings', Oxford Economic Papers 57, 191-261.

da Costa, C. E. \& Maestri, L. J. (2007), 'The risk properties of human capital and the design of government policies', European Economic Review 51, 695-713.

Diamond, P. A. (2006), 'Comment on "New Dynamic Public Finance: A User's Guide"', NBER Macroeconomic Annual pp. 365-379.

Diamond, P. \& Mirrlees, J. (1978), 'A model of optimal social insurance with variable retirement', Journal of Public Economics 10, 295-336.

Eaton, J. \& Rosen, H. S. (1980), 'Taxation, human capital, and uncertainty', American Economic Review 70, 705-715.

Golosov, M., Kocherlakota, N. \& Tsyvinsk, A. (2003), 'Optimal indirect and capital taxation', Review of Economic Studies 70, 569-587. 
Golosov, M. \& Tsyvinski, A. (2007), 'Optimal taxation with endogenous insurance markets', Quartely Journal of Economics 122, 487-534.

Golosov, M., Tsyvinski, A. \& Werning, I. (2006), 'New dynamic public finance: A user's guide', NBER Macroeconomic Annual pp. 317-363.

Grochulski, B. \& Piskorski, T. (2006), 'Risky human capital and deferred capital income taxation', Federal Reserve Bank of Richmond Working Paper No. 06-13.

Guesnerie, R. \& Seade, J. (1982), 'Nonlinear pricing in a finite economy', Journal of Public Economics 17, 157-180.

Hamilton, J. (1987), 'Optimal wage and income taxation with wage uncertainty', International Economic Review 28, 373-388.

Jacobs, B. \& Bovenberg, L. (2008), 'Optimal taxation of human capital and the earnings function', CESifo Working Paper No. 2250.

Jacobs, B., Schindler, D. \& Yang, H. (2008), 'Optimal taxation of risky human capital', Erasmus University Rotterdam, Mimeo.

Levhari, D. \& Weiss, Y. (1974), 'The effects of risk on the investment in human capital', American Economic Review 64, 950-963.

Maldonado, D. (2008), 'Education policies and optimal taxation', International Tax and Public Finance 15, 131-143.

Mirrlees, J. (1971), 'An exploration in the theory of optimum income taxation', Review of Economic Studies 38, 175-208.

Werning, I. (2007), 'Optimal fiscal policy with redistribution', Quartely Journal of Economics 122, 925-967. 


\section{Appendix}

\section{First Order Conditions for the Case with Unobservable Education}

Here we report the first order conditions for the planner's problem (25). The first order conditions for $c_{0}(\mu)$ can be written as

$$
u^{\prime}\left(c_{0}(\mu)\right)\left\{\left(1+\sum_{\sigma \in \Sigma} \phi_{\sigma}\right)-\sum_{\sigma \in \Sigma} \sum_{\mu^{\prime} \in I_{\mu}^{\sigma}} \phi_{\sigma} \frac{\pi\left(\mu^{\prime}\right)}{\pi(\mu)} \frac{u^{\prime}\left(c_{0}(\mu)-w_{0} \Delta_{z}(\sigma)\right)}{u^{\prime}\left(c_{0}(\mu)\right)}\right\}=\lambda .
$$

The first order condition for $c_{1}(\mu, \theta)$ can be written as

$$
u^{\prime}\left(c_{1}(\mu, \theta)\right)\left\{\left(1+\sum_{\sigma \in \Sigma} \phi_{\sigma}\right)-\sum_{\sigma \in \Sigma} \sum_{\left(\mu^{\prime}, \theta^{\prime}\right) \in I_{(\mu, \theta)}^{\sigma}} \phi_{\sigma} \frac{\pi\left(\mu^{\prime}, \theta^{\prime}\right)}{\pi(\mu, \theta)}\right\}=\frac{\lambda}{\beta} .
$$

The first order condition for $y_{1}(\mu, \theta)$ can be written as

$\frac{v^{\prime}\left(l_{1}(\mu, \theta)\right)}{w(z, \theta)}\left\{-\left(1+\sum_{\sigma \in \Sigma} \phi_{\sigma}\right)+\sum_{\sigma \in \Sigma} \sum_{\left(\mu^{\prime}, \theta^{\prime}\right) \in I_{(\mu, \theta)}^{\sigma}} \phi_{\sigma} \frac{\pi\left(\mu^{\prime}, \theta^{\prime}\right)}{\pi(\mu, \theta)} \frac{v^{\prime}\left(l_{1}^{\sigma}\left(\mu^{\prime}, \theta^{\prime}\right)\right)}{v^{\prime}\left(l_{1}(\mu, \theta)\right)} \frac{w(z, \theta)}{w\left(z+\Delta_{z}(\sigma), \theta^{\prime}\right)}\right\}=\frac{\lambda}{\beta}$.

Finally, the first order condition for $z$ can be written as

$$
\begin{aligned}
\frac{\lambda}{\beta} w_{0}= & \sum_{(\mu, \theta) \in M \times \Theta} \pi(\mu, \theta)\left\{-\left(1+\sum_{\sigma \in \Sigma} \phi_{\sigma}\right) v^{\prime}\left(l_{1}(\mu, \theta)\right) l_{1}(\mu, \theta) \frac{w_{z}(z, \theta)}{w(z, \theta)}\right. \\
& \left.+\sum_{\sigma \in \Sigma} \phi_{\sigma} v^{\prime}\left(l_{1}^{\sigma}(\mu, \theta)\right) l_{1}^{\sigma}(\mu, \theta) \frac{w_{z}\left(z+\Delta_{z}(\sigma), \theta\right)}{w\left(z+\Delta_{z}(\sigma), \theta\right)}\right\} .
\end{aligned}
$$

\section{First Order Conditions for the Case with Unobservable Consumption}

Here we report the first order conditions for the planner's problem (31). The first order conditions for $x_{0}(\mu)$ can be written as

$$
u^{\prime}\left(x_{0}(\mu)\right)\left\{\left(1+\sum_{\sigma \in \Sigma} \phi_{\sigma}\right)-\sum_{\sigma \in \Sigma} \sum_{\mu^{\prime} \in I_{\mu}^{\sigma}} \phi_{\sigma} \frac{\pi\left(\mu^{\prime}\right)}{\pi(\mu)} \frac{u^{\prime}\left(c_{0}^{\sigma}\left(\mu^{\prime}\right)\right)}{u^{\prime}\left(x_{0}(\mu)\right)}\right\}=\lambda-\rho_{\mu} u^{\prime \prime}\left(x_{0}(\mu)\right) .
$$

The first order conditions for $x_{1}(\mu, \theta)$ can be written as

$u^{\prime}\left(x_{1}(\mu, \theta)\right)\left\{\left(1+\sum_{\sigma \in \Sigma} \phi_{\sigma}\right)-\sum_{\sigma \in \Sigma} \sum_{\left(\mu^{\prime}, \theta^{\prime}\right) \in I_{\mu, \theta}^{\sigma}} \phi_{\sigma} \frac{\pi\left(\mu^{\prime}, \theta^{\prime}\right)}{\pi(\mu, \theta)} \frac{u^{\prime}\left(c_{1}^{\sigma}\left(\mu^{\prime}, \theta^{\prime}\right)\right)}{u^{\prime}\left(x_{1}(\mu, \theta)\right)}\right\}=\frac{\lambda}{\beta}+\rho_{\mu} R u^{\prime \prime}\left(x_{1}(\mu, \theta)\right)$, 
where $c_{1}^{\sigma}(\mu, \theta)$ is the period-1 consumption at node $(\mu, \theta)$ of an agent who chooses reporting strategy $\sigma \in \Sigma$. The first order conditions for $y_{1}(\mu, \theta)$ and $z$ can be written as in A3 and A4 but with $\Delta_{z}(\sigma) \equiv 0$. The first order condition for $R^{*}$ is given in equation (33). 

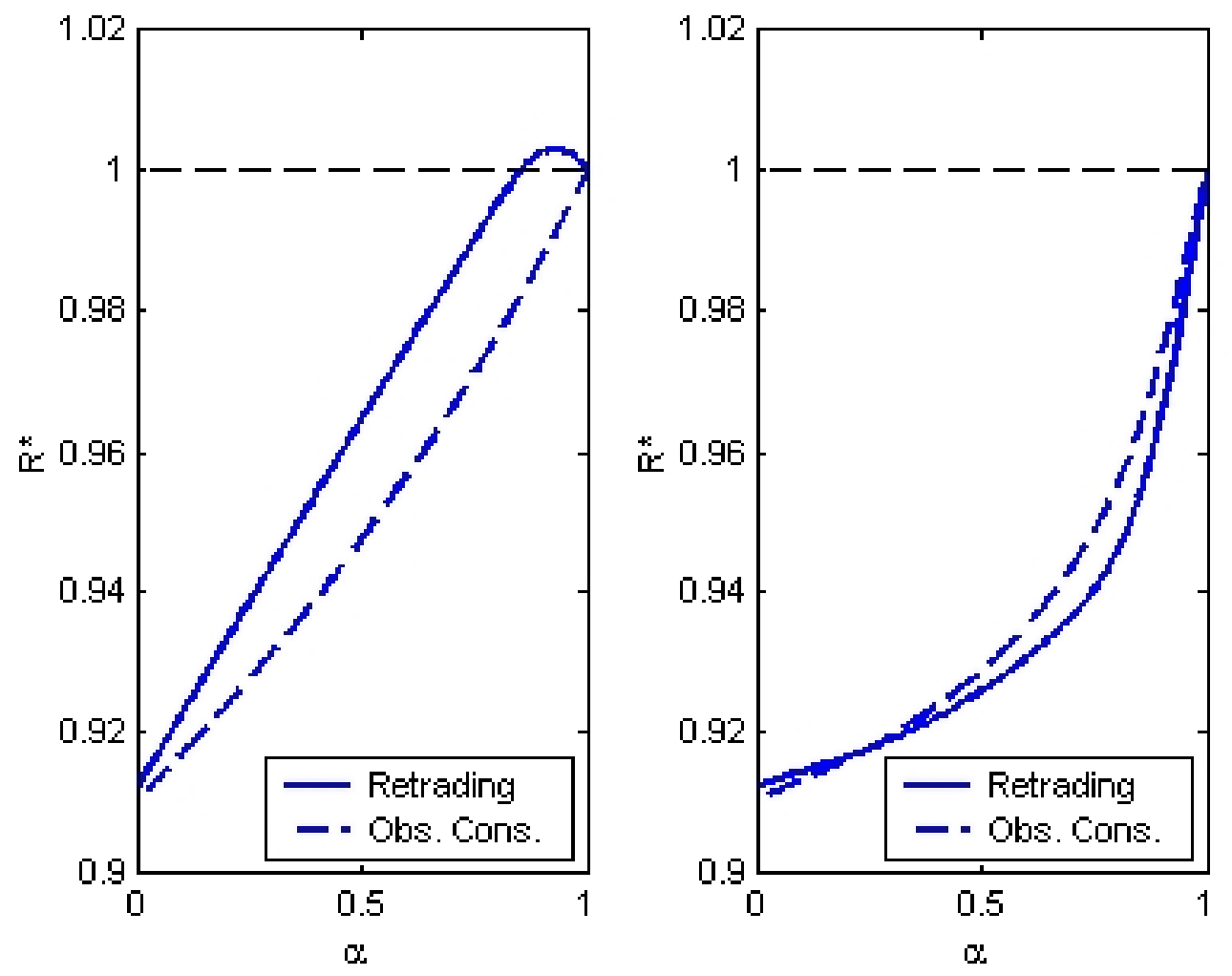

Figure 1: The optimal $R$ when a fraction $\alpha$ of those individuals who obtain a favorable productivity shock (left panel), or a fraction $\alpha$ of those individuals who obtain an unfavorable productivity shock (right panel), learn this prior to completing their consumption plans. 